\title{
Health Literacy Gaps in Online Resources for Cirrhotic Patients
}

\author{
Trisha Kaundinya ${ }^{\text {a }}$, Nikhilesh Mazumder ${ }^{\text {a, b }}$, Kofi Atiemo ${ }^{\text {a, c }}$, Ari Spellman ${ }^{\text {a, d }}$, \\ Amna Daud $^{\text {a }}$, Laura Marie Curtis ${ }^{\mathrm{e}}$, Daniela Patricia Ladner ${ }^{\mathrm{a}}$, f, $\mathrm{g}$
}

\begin{abstract}
Background: The average readability level in the USA is a sixth grade level and for patients with chronic disease it is lower. Cirrhosis is a prevalent chronic disease that requires complex knowledge and instructions to manage. No research has been done about the understandability of online educational content for cirrhotic patients. Patients can find online materials curated by both general health platforms and high-volume liver transplant centers, and thus these materials were analyzed.
\end{abstract}

Methods: After determining exclusion criteria, the websites of the top 20 general health platform results and the websites of the top 20 high-volume hepatology centers were analyzed. Readability was assessed using the Patient Education Materials Assessment Tool (Audiovisual Materials) (PEMAT-A/V), Flesch-Kincaid Grade Level tests, word counts, sentence counts, words per sentence, and time for an average sixth grader to read.

Results: The mean grade level readabilities were 12.3 and 11.3 for the general resources and the transplant center resources, respectively. The online resources ranged from 9 to 389 sentences requiring an average of $9.8 \mathrm{~min}$ to read. The mean PEMAT-A/V scores were $70.05 \%$ for the general resources and $72.45 \%$ for the transplant center resources. There was a statistically significant difference in the Flesch-Kincaid grade level, sentence number, words per sentence, word count, and time for an average sixth grader to read the general

Manuscript submitted February 14, 2020, accepted April 10, 2020

aNorthwestern University Transplant Outcomes Research Collaborative (NUTORC), Comprehensive Transplant Center, Feinberg School of Medicine, Northwestern University, Chicago, IL, USA

bDivision of Gastroenterology, Northwestern Medicine, Chicago, IL, USA

'Division of Transplant, Department of Surgery, Tulane University, New Orleans, LA, USA

dUniversity of Michigan Medical School, Ann Arbor, MI, USA

${ }^{\mathrm{e}}$ Health Literacy and Learning Program (HeLP), Division of General Internal Medicine and Geriatrics, Feinberg School of Medicine, Chicago, IL, USA fDivision of Transplantation, Northwestern Medicine, Chicago, IL, USA gCorresponding Author: Daniela P. Ladner, Northwestern University Transplant Outcomes Research Collaborative (NUTORC), Comprehensive Transplant Center, Feinberg School of Medicine, Northwestern University, 676 North St. Clair Street, Suite 1900, Chicago, IL 60611, USA.

Email: dladner@nm.org

doi: https://doi.org/10.14740/jcs401 resources and transplant center resources $(\mathrm{P}<0.05)$.

Conclusions: The online resources both from health platforms and hepatology centers available to patients with cirrhosis are too long and complex and underscore the need for simpler and shorter resources.

Keywords: Patient education; Cirrhosis; Media and social media; Quality of life

\section{Introduction}

An estimated five million people suffer from liver cirrhosis, a chronic disease that requires extensive health care resources and intricate understanding to obtain and follow optimal treatment, such as regular care through gastroenterologists, liver cancer screening through ultrasounds, and endoscopies to assess portal hypertension [1]. However, disease understanding in this population is generally lacking. In Denmark, cirrhotic patients were found to have low levels of understanding about their conditions [2]. Another study in Melbourne identified that hepatitis B patients had significant gaps in their knowledge of the disease, transmission routes, and treatment [3]. In a US query of patients with liver cirrhosis, $15 \%$ listed that their most important disease-related need is accessible and understandable educational material [4].

Patient education materials can promote understanding [5]. Most patients of all socioeconomic and age strata seek information about their disease online, either through general internet resources or attached to large medical centers [6]. But online resources often tower above patients' reading levels, which average at sixth grade [7]. Educational material can also be filled with medical jargon [8]. One US study reported that online reading materials on hepatitis $\mathrm{B}$, hepatitis $\mathrm{C}$, hepatocellular carcinoma, and cirrhosis had a mean reading level over 10 th grade [9]. Similar challenges in patient reading materials have been described for kidney transplantation and other chronic conditions such as diabetes $[10,11]$.

Little is known about the comprehensibility of the highest visibility online resources on liver cirrhosis. Over $95 \%$ of traffic from an online search comes from the first page of results, so the first few web links have the greatest chance of being read by patients [12]. In this study, we therefore aimed to assess the understandability of the top patient education materials about 
liver cirrhosis available online through general search terms as well as the websites of large hepatology centers.

\section{Materials and Methods}

This research did not require human subjects, and Institutional Review Board (IRB) review was not required. Two online searches were performed. One online search for "liver cirrhosis" was performed using the "Google" search engine ("Google"). The second search targeted the highest volume liver transplant centers ("hepatology centers"). For the "Google" search, the websites of medical centers, news articles, magazine articles, and videos were excluded as medical center websites were analyzed in the second search, and patients would have to listen to videos or download issues of magazines to get information (they were not quick readable sources). The results from the remaining top 20 websites were analyzed. For the "hepatology centers" search, the highest volume liver transplant centers were determined through the United Network for Organ Sharing (UNOS). The top 20 centers with websites on liver cirrhosis were analyzed. For both searches, the grade level, word count, number of sentences, and number of words per sentence were calculated. Grade level readability was determined using the Flesch-Kincaid grade level analyzer, which takes into account word length and sentence length. Averages and standard deviations were calculated. The time needed to read each passage was calculated based on an average sixth grade reading speed of 140 words/min as in Hasbrouck and Tindal's norms [13]. In addition to a grade level metric of readability, the Patient Education Materials Assessment Tool (Audiovisual Materials) (PEMAT-A/V) test on understandability was used by two raters to evaluate all 40 samples. This test included subsections on "Content", "Word Choice and Style", "Organization", "Layout and Design", and "Visual Aids" [14]. The search was performed on February 1, 2019. A two-tailed paired $t$-test for means was used with $\alpha=0.05$ level of significance to compare the metrics of Flesch-Kincaid grade level, sentence number, words per sentence, word count, time for an average sixth grader to read, and PEMAT-A/V score for the "Google" search and "hepatology centers" search.

\section{Results}

\section{Google}

After exclusion of search results from medical centers (12) (see second analysis), videos (1), and newspaper and magazine articles (4), the top 20 search results were analyzed. The mean grade level readability was $12.3(6.8$ - 20.8), the mean word count was $1,841(200-7,767)$, the mean number of sentences was $105(9-389)$, and the mean number of words per sentence was 19.6 (10.6 - 29.4) (Table 1). Sixteen (80\%) websites' resources had subsections on etiology, symptoms, and treatments, which were included in the analysis, while four $(20 \%)$ websites had no information on these subsections. The Medline Plus web resource had the lowest reading grade level at 6.8, with the lowest words per sentence (10.6) and fifth lowest PEMAT score (61). The most complicated web resource was the National Center for Biotechnology Information (NCBI) with a readability at 20.8 grade and a PEMAT score of 64 , which was the second lowest score. The average time for a sixth grader to read these web resources was $13.15 \mathrm{~min}(1.4-21.9)$. The average PEMAT score for this sample was 70.05 (50 - 93).

\section{Hepatology centers}

To find information on 20 websites of high-volume hepatology centers, 35 were queried. Of the 20 centers with online information on cirrhosis, five were in the Northeastern USA, four on the West Coast, six in the Midwest, and five in the South; 19 out of $20(95 \%)$ were academic institutions. The 20 centers performed an annual average of 122.9 (93 - 170) liver transplants from 2017 to 2018 . Among the 20 online texts, the mean grade level readability was $11.3(6.7-17.2)$, the mean word count was 904 (276 - 2,677), the mean number of sentences was 64.15 (12 - 194), and the mean number of words per sentence was 18 (11 - 32) (Table 2). Subsections related to etiology, symptoms, and treatments were found on $18(90 \%)$ websites. University of Wisconsin's patient materials had the lowest reading grade level at 6.7, and Georgetown Medical Hospital's patient materials had the highest reading level at 17.2. However, Georgetown's materials had a higher PEMAT score (80) than University of Wisconsin (64). Ohio State University's patient materials had the lowest number of words (175) and would take a sixth grade reader the shortest number of minutes to read (1.3), while the readability was at a 10.3 grade level and the PEMAT score was 73. The average time for a sixth grader to read these passages was $6.5 \min (1.3$ - 19.1). The average PEMAT score for this sample was $72.45 \%(40-90 \%)$.

\section{Comparison between centers}

The readability of the top 20 sources found through both a general web search and a high-volume hepatology center search was over an 11 th grade level, with transplant centers providing materials an average of one grade lower than general resources (11.3 vs. 12.3, respectively). In addition to Flesch-Kincaid grade level, the average number of sentences, words per sentence, word count, and time for an average sixth grader were higher for the general resources than the transplant center resources (105 vs. $64.15,19.63$ vs. $18.02,1,841$ vs. $904,13.15$ vs. 6.46 , respectively). A two-tailed paired $t$-test for means showed a statistically significant difference in the average Flesch-Kincaid grade level, sentence number, words per sentence, word count, and time for an average sixth grader to read the general resources and transplant center resources $(\mathrm{P}<0.05)$ (Fig. 1).

\section{Discussion}

The web sources readily available to patients with liver cirrhosis required an average reading level over 11 th grade and the 
Table 1. Readability and Understandability of Top "Google" Searches

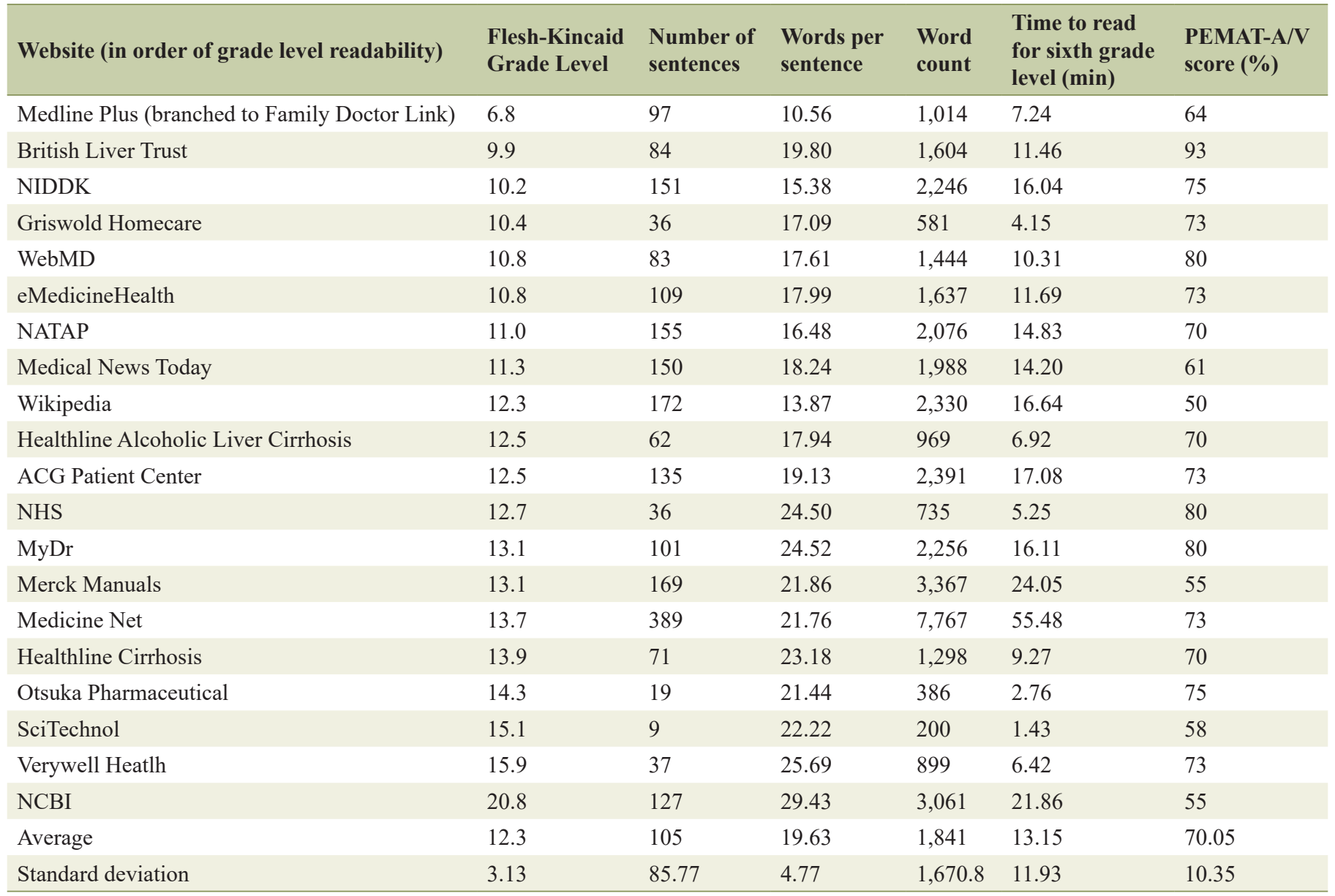

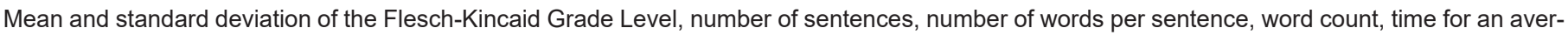

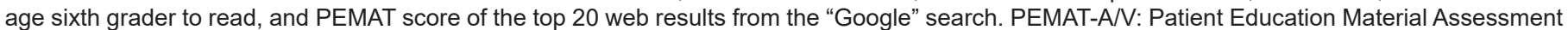

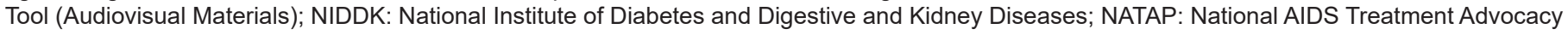
Project; ACG: American College of Gastroenterology; NHS: National Health Service; NCBI: National Center for Biotechnology Information.

ability for a sixth grader to focus for more than $9.8 \mathrm{~min}$. In the USA the average adult reading proficiency is at eighth grade, but over $20 \%$ of patients read below a fifth grade level $[7,15]$. Hence, the reading level required to read about cirrhosis far exceeds the national average reading level and far more exceeds the reading level of chronically ill patients. In a study of asthma patients, over $40 \%$ read at or below a sixth grade level despite being high school graduates [16].

In addition to high reading grade levels, the average PEMAT understandability score was 71.25 , with transplant centers providing slightly more understandable materials than general web resources (72.45 vs. 70.05, respectively) (Fig. 1). Only four websites had a score above 90 in understandability and one was as low as 40. A patient with liver cirrhosis will likely struggle to comprehend any significant amount of online information provided when it is, on average, $70 \%$ understandable. While there is not an average educational website score to reference for the PEMAT, trends were observed that lowered scores of the analyzed web results. More than half of websites in both searches did not receive points in the PEMAT subsec- tions titled "Word Choice and Style" and "Layout and Design", because they used passive voice and did not use a readable text size. Both of these factors have been independently linked to the capacity to remember words and comprehend text $[17,18]$. Over $75 \%$ of all websites did not use any images, which have been shown to facilitate recall of main ideas of text [19]. Websites with lower grade level readabilities did not have greater understandability scores than websites with higher grade level readabilities, which has also been observed in an analysis of diabetes-related education materials [20].

As of 2019, the duration of an average web user's Google search session was under 9 min [21]. While some cirrhosis websites might take under $9 \mathrm{~min}$ to read, they are unlikely to be understood because cirrhotic patients are not average web users. Many suffer from hepatic encephalopathy, which causes neurocognitive impairment, issues with executive function, slower problem solving, and attention deficits. Hence, they need more time to read long passages on websites with small fonts and few images [22]. They also have a decreased ability to sustain focus while reading which limits their capacity and 
Table 2. Readability and Understandability of Top "Hepatology Centers" Searches

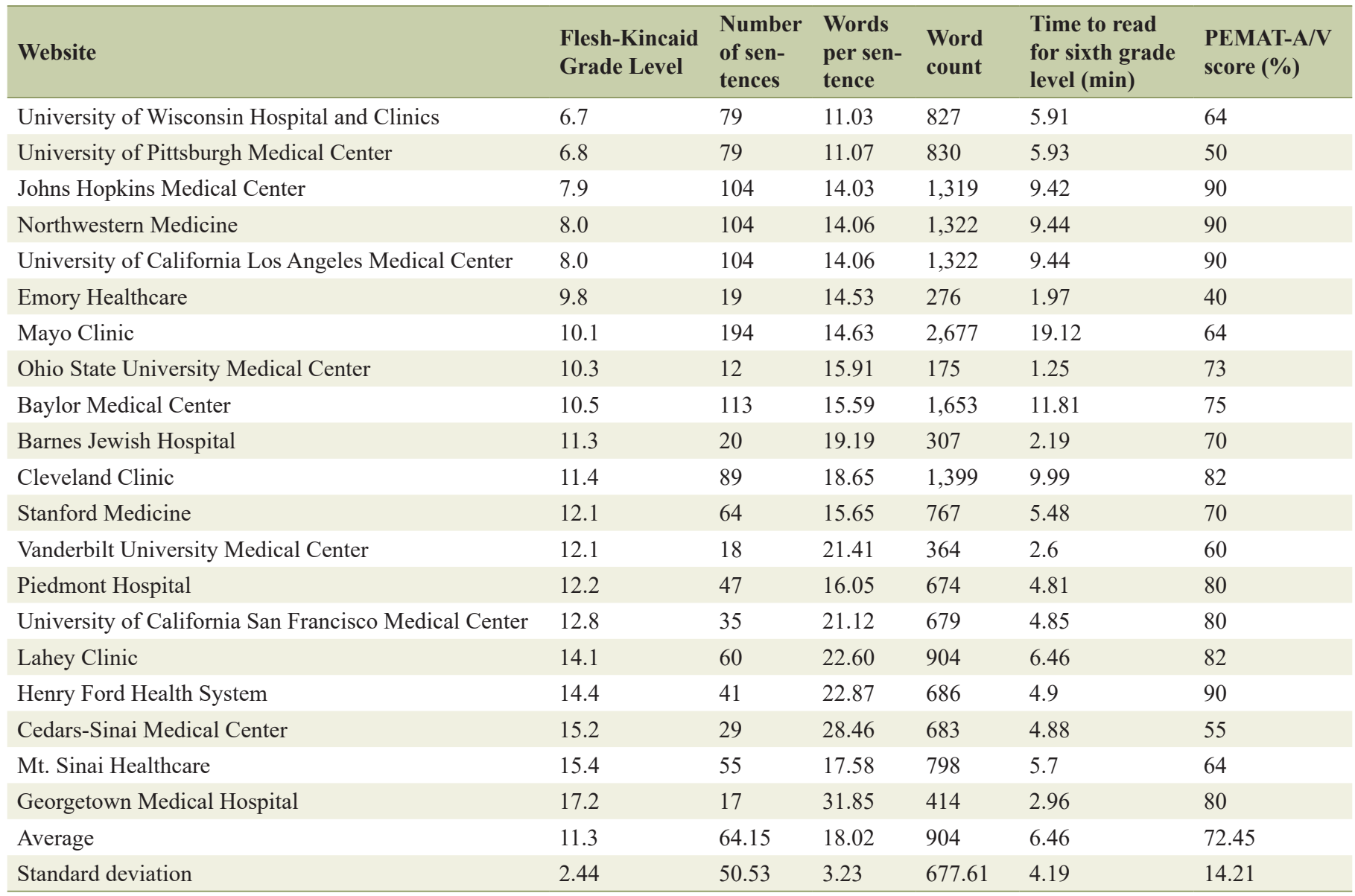

Mean and standard deviation of the Flesch-Kincaid Grade Level, number of sentences, number of words per sentence, word count, time for an average sixth grader to read, and PEMAT score of the top 20 web results from the "Hepatology Centers" search. PEMAT-A/V: Patient Education Material Assessment Tool (Audiovisual Materials).

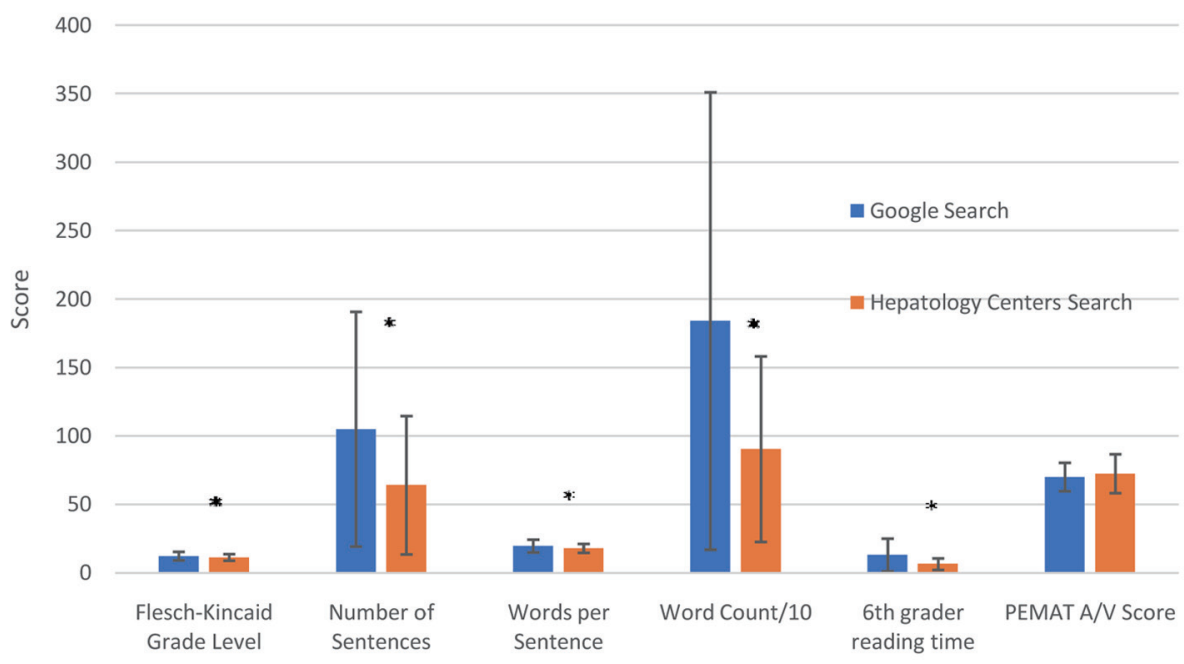

Figure 1. Comparison between "Google" search and "Hepatology Centers" search. Comparison of mean ( \pm SD) scores between "Google" search results and results from "Hepatology Centers" online searches. *P < 0.05. PEMAT-A/V: Patient Education Material Assessment Tool (Audiovisual Materials); SD: standard deviation. 
motivation to learn about cirrhosis [23].

Improving the readability and comprehensibility of patient education materials has proven to be successful at improving patient understanding in other contexts $[24,25]$. Cirrhotic patients face fatigue, a lower quality of life, and helplessness and could potentially gain a better sense of control by understanding their disease [26]. Lower levels of disease understanding in cirrhotic patients have shown to negatively influence selfmanagement behaviors, while well-informed cirrhotic patients are more likely to be adherent to their complex and exhausting treatment regimens, which leads to greater well-being and less fatigue [27-30].

This study was limited to reading resources and excluded other sources such as locally available paper pamphlets provided by centers or videos. The analyses were performed using the Flesh-Kincaid Grade Level tool to measure readability and PEMAT to measure understandability. There are other tools (e.g. Gunning Fog Index, SMOG score) available, but cursory application did not change the reported results.

Educational material is too long and complex for the target population. Involving patients with cirrhosis in creating appropriate educational material that is available online through simple searches might significantly improve understanding of this chronic disease and may increase guideline adherence and satisfaction [31].

\section{Conclusions}

Our findings corroborate the claim of liver cirrhosis patients in a national online query indicating that information about the disease is elusive, as most general websites and especially those from hepatology centers are too high in grade level readability, very long (13 min for the Google search and $6.5 \mathrm{~min}$ for hepatology centers) and low in understandability $(70.05 \%$ for the Google search and $72.45 \%$ for the hepatology centers) (Fig. 1) [4]. No websites contained information that was a sixth grade level and had a 90\% PEMAT score. This obvious educational resource gap can easily be mitigated through targeted information made available and is likely to positively affect treatment adherence of patients with liver cirrhosis [32].

\section{Acknowledgments}

None to declare.

\section{Financial Disclosure}

PCORI-Tier 3: National patient community, caregiver and advocacy group directed strategies to lead comparative effectiveness research for liver cirrhosis (PI: Daniela P. Ladner).

\section{Conflict of Interest}

None to declare.

\section{Informed Consent}

\author{
Not applicable.
}

\section{Author Contributions}

TK contributed to data analysis/interpretation, drafting article, critical revision of article, statistics analysis; NM, KA and LMC contributed to the critical revision of article; AS contributed to data analysis/interpretation; AD contributed to data analysis/interpretation, and critical revision of article; DPL contributed to concept/design, and critical revision of article. All authors approved the article.

\section{Data Availability}

The authors declare that data supporting the findings of this study are available within the article.

\section{References}

1. Traussnigg S, Kienbacher C, Halilbasic E, Rechling C, Kazemi-Shirazi L, Hofer H, Munda P, et al. Challenges and management of liver cirrhosis: practical issues in the therapy of patients with cirrhosis due to NAFLD and NASH. Dig Dis. 2015;33(4):598-607.

2. Freundlich Grydgaard M, Bager P. Health literacy levels in outpatients with liver cirrhosis. Scand J Gastroenterol. 2018;53(12):1584-1589.

3. Dahl TF, Cowie BC, Biggs BA, Leder K, MacLachlan JH, Marshall C. Health literacy in patients with chronic hepatitis B attending a tertiary hospital in Melbourne: a questionnaire based survey. BMC Infect Dis. 2014;14:537.

4. Ladner DP. P2P liver cirrhosis study. PCOR Net. 2018.

5. DeMarco J, Nystrom M, Salvatore K. The importance of patient education throughout the continuum of health care. Journal of Consumer Health on the Internet. 2011;15(1):22-31.

6. Bergeron B. Online patient-education options. MedGenMed. 2004;6(2):54.

7. Davis TC, Crouch MA, Wills G, Miller S, Abdehou DM. The gap between patient reading comprehension and the readability of patient education materials. J Fam Pract. 1990;31(5):533-538.

8. Hansberry DR, Agarwal N, John ES, John AM, Agarwal P, Reynolds JC, Baker SR. Evaluation of internet-based patient education materials from internal medicine subspecialty organizations: will patients understand them? Intern Emerg Med. 2017;12(4):535-543.

9. Gulati R, Nawaz M, Pyrsopoulos NT. Comparative analysis of online patient education material pertaining to hepatitis and its complications. Eur J Gastroenterol Hepatol. 2016;28(5):558-566.

10. Boulos MN. British internet-derived patient information on diabetes mellitus: is it readable? Diabetes Technol 
Ther. 2005;7(3):528-535.

11. Zhou EP, Kiwanuka E, Morrissey PE. Online patient resources for deceased donor and live donor kidney recipients: a comparative analysis of readability. Clin Kidney J. 2018;11(4):559-563.

12. Kaye L. 95 percent of web traffic goes to sites. Google SERPs [study]. 2014:1

13. Hasbrouck J, Tindal G. Oral reading fluency norms: a valuable assessment tool for reading teachers. Reading Teacher - READ TEACH. 2006;59:636-644.

14. Shoemaker SJ, Wolf MS, Brach C. Development of the Patient Education Materials Assessment Tool (PEMAT): a new measure of understandability and actionability for print and audiovisual patient information. Patient Educ Couns. 2014;96(3):395-403.

15. Morony S, Flynn M, McCaffery KJ, Jansen J, Webster AC. Readability of Written Materials for CKD Patients: A Systematic Review. Am J Kidney Dis. 2015;65(6):842-850.

16. Williams MV, Baker DW, Honig EG, Lee TM, Nowlan A. Inadequate literacy is a barrier to asthma knowledge and self-care. Chest. 1998;114(4):1008-1015.

17. Halamish V, Nachman H, Katzir T. The Effect of Font Size on Children's Memory and Metamemory. Front Psychol. 2018;9:1577.

18. Shapiro J. An investigation into the effect of active and passive voice on the comprehension of seventh grade good and poor readers. New York: Education, State University College at Brockport. 1982.

19. Haring MJ, Fry MA. Effect of pictures on children's comprehension of written text. ECTJ. 1979;27(3):185-190.

20. Lipari M, Berlie H, Saleh Y, Hang P, Moser L. Understandability, actionability, and readability of online patient education materials about diabetes mellitus. Am J Health Syst Pharm. 2019;76(3):182-186.

21. Facebook SL. Google lose share of time spent on-site as bing sees increase. 2019. https://www.mediapost.com/ publications/article/336323/facebook-google-lose-shareof-time-spent-on-site.html.

22. Bajaj JS, Wade JB, Sanyal AJ. Spectrum of neurocognitive impairment in cirrhosis: Implications for the assessment of hepatic encephalopathy. Hepatology. 2009;50(6):20142021.

23. Liu CF, Kuo KM. Does information overload prevent chronic patients from reading self-management educational materials? Int J Med Inform. 2016;89:1-8.

24. Baker GC, Newton DE, Bergstresser PR. Increased readability improves the comprehension of written information for patients with skin disease. J Am Acad Dermatol. 1988;19(6):1135-1141.

25. Overland JE, Hoskins PL, McGill MJ, Yue DK. Low literacy: a problem in diabetes education. Diabet Med. 1993;10(9):847-850.

26. Loria A, Escheik C, Gerber NL, Younossi ZM. Quality of life in cirrhosis. Curr Gastroenterol Rep. 2013;15(1):301.

27. Mackey LM, Doody C, Werner EL, Fullen B. Selfmanagement skills in chronic disease management: what role does health literacy have? Med Decis Making. 2016;36(6):741-759.

28. Serper M, Patzer RE, Reese PP, Przytula K, Koval R, Ladner DP, Levitsky J, et al. Medication misuse, nonadherence, and clinical outcomes among liver transplant recipients. Liver Transpl. 2015;21(1):22-28.

29. Miller TA. Health literacy and adherence to medical treatment in chronic and acute illness: A meta-analysis. Patient Educ Couns. 2016;99(7):1079-1086.

30. Hayward KL, Valery PC, Martin JH, Karmakar A, Patel PJ, Horsfall LU, Tallis CJ, et al. Medication beliefs predict medication adherence in ambulatory patients with decompensated cirrhosis. World J Gastroenterol. 2017;23(40):7321-7331.

31. Allison R, Evans PH, Kilbride C, Campbell JL. Secondary prevention of stroke: using the experiences of patients and carers to inform the development of an educational resource. Fam Pract. 2008;25(5):355-361.

32. Polis S, Zang L, Mainali B, Pons R, Pavendranathan G, Zekry A, Fernandez R. Factors associated with medication adherence in patients living with cirrhosis. J Clin Nurs. 2016;25(1-2):204-212. 